\title{
Association of Antigen-Stimulated Release of Tumor Necrosis Factor-Alpha in Whole Blood with Response to Chemotherapy in Patients with Pulmonary Multidrug-Resistant Tuberculosis
}

\author{
Seok-Yong Eum ${ }^{a}$ Ye-Jin Lee ${ }^{a}$ Jin-Hong Min ${ }^{b}$ Hyun-Kyung Kwak ${ }^{a}$ \\ Min-Sun Hong ${ }^{a}$ Ji-Hye Kong ${ }^{a}$ Soo-Hee Hwang ${ }^{b}$ Seung-Kyu Park ${ }^{b}$ \\ Jason J. LeBlanc ${ }^{d}$ Laura E. Viad Clifton E. Barry, III ${ }^{d}$ Sang-Nae Cho ${ }^{c}$ \\ a Divisions of Immunopathology and Cellular Immunology, and of Molecular Microbiology, International \\ Tuberculosis Research Center, ${ }^{\mathrm{b}}$ National Masan Tuberculosis Hospital, Masan, and ' Department of Microbiology, \\ Yonsei University College of Medicine, Seoul, Republic of Korea; ${ }^{\mathrm{d}}$ Tuberculosis Research Section, National Institute \\ of Allergy and Infectious Diseases, National Institutes of Health, Bethesda, Md., USA
}

\section{Key Words}

Chemotherapy $\cdot$ Interferon- $\gamma \cdot$ Interleukin-10 •

Interleukin-12 Multidrug-resistant tuberculosis .

Mycobacterial clearance $\cdot$ Tuberculosis $\cdot$ Tumor necrosis factor- $\alpha$

\begin{abstract}
Background: We have previously reported that TNF- $\alpha$ levels correlate to total mycobacterial burden in tuberculosis (TB) patients. Objective: To characterize the dynamics of cytokine responses in TB patients during chemotherapy to identify potential surrogate markers for effective treatment. Methods: Following induction by culture filtrate proteins in whole blood, production patterns of TNF- $\alpha$, IL-10, IFN- $\gamma$ and IL-12 were measured in 23 non-multidrug-resistant (MDR)-TB and 16 MDR-TB patients and in 31 healthy controls. Rates of mycobacterial clearance from the sputum were then measured and compared. Results: Prior to the initiation of chemotherapy, TNF- $\alpha$ and IL-10 levels were significantly higher in TB patients than in healthy controls while IFN- $\gamma$ and IL-12
\end{abstract}

levels were similar. During chemotherapy, the levels of all 4 cytokines increased. We evaluated these responses separately in patients that did and did not clear their sputum culture at 2 and 6 months. At 2 months, decreases in both IFN- $\gamma$ and IL-12 correlated strongly with a successful early response, while after 6 months of therapy, when half (7/14) of MDR-TB patients were still sputum culture positive, downregulation of TNF- $\alpha$ was uniquely correlated with sputum conversion between the groups. Conclusion: Our findings suggest the possibility that the regulation of TNF- $\alpha$ production in whole blood may be a more specific indicator of sputum conversion at 6 months than IFN- $\gamma$, IL-12 or IL-10 in MDR-TB patients.

Copyright $\odot 2010$ S. Karger AG, Basel

\section{Introduction}

Mycobacterium tuberculosis (Mtb) is the causative agent of pulmonary tuberculosis (TB). Currently, there are two major problems facing the medical community

\section{KARGER}

(C) 2010 S. Karger AG, Basel

Fax +4161306 1234 E-Mail karger@karger.ch www.karger.com 
Table 1. Sputum culture AFB-negative conversion rate in non-MDR and MDR-TB patients along with therapy

\begin{tabular}{|c|c|c|c|c|c|c|}
\hline \multirow[t]{3}{*}{ Patients enrolled } & \multicolumn{6}{|c|}{ Sputum culture AFB } \\
\hline & \multicolumn{2}{|c|}{ non-MDR-TB $(\mathrm{n}=23)$} & \multicolumn{2}{|c|}{ MDR-TB $(n=16)$} & \multicolumn{2}{|c|}{ total $(\mathrm{n}=39)$} \\
\hline & - & + & - & + & - & + \\
\hline At enrollment & $12(54.5 \%)$ & $10(45.5 \%)$ & $0(0 \%)$ & $16(100 \%)$ & $12(31.6 \%)$ & $26(68.4 \%)$ \\
\hline After 2 months & $21(95.5 \%)$ & $1(4.5 \%)$ & $2(12.5 \%)$ & $14(87.5 \%)$ & $23(60.5 \%)$ & $15(39.5 \%)$ \\
\hline After 6 months & $22(100 \%)$ & $0(0.0 \%)$ & $8(50.0 \%)$ & $8(50.0 \%)$ & $30(78.9 \%)$ & $8(21.1 \%)$ \\
\hline
\end{tabular}

One of the 39 patients enrolled who was culture negative at enrollment and not responsive to therapy was omitted from the analysis.

concerning the fight against Mtb. First, the increasing emergence of multidrug-resistant (MDR)-TB has rendered common therapies against the bacteria ineffective. Second, the current testing methodologies for the diagnosis of TB are extremely slow. Because Mtb is a slowgrowing organism, it can take up to 8 weeks for an accurate $\mathrm{Mtb}$ diagnosis to be made. As a result, there is an increasing need for both novel therapies and rapid diagnostic methods.

After Mtb infection, healthy individuals develop a cellular immune response, which arrests the growth of the microorganism, preventing progression to clinical TB $[1$, 2]. However, susceptible individuals develop pulmonary TB. Upon inhalation of aerosolized Mtb, the pathogen is engulfed by macrophages. Once inside the phagosome, virulent Mtb inhibits the acidification of the compartment [3]. This inhibition allows the bacilli to reside within the macrophage, sequestered away from the bactericidal and antigen-generating machinery of the cell. As a result, Mtb establishes a safe and long-term resting area for replication, which eventually results in cell lysis and bacterial spread.

Upon stimulation by a pathogen, macrophages engulf the offending particle, and upon its destruction, they present smaller peptide antigens on their surface. These antigens are then recognized by Th1 cells, which in turn secrete various cytokines, including IFN- $\gamma$, IL-12 and TNF- $\alpha$. These cytokines in turn activate resting macrophages, which trigger the immune response. In order to suppress the immune response once an infection has been cleared, and to prevent autoimmune responses to self-antigens, other cytokines downregulate the immune system. IL-10 activates B and Th2 cells while inhibiting Th1 cytokine production. Activated Th2 cells secrete IL-
4, which also inhibits Th1 cell activity and inactivates macrophages.

Previously, it has been reported that Mtb stimulation of peripheral blood mononuclear cells (PBMCs) isolated from TB patients resulted in a diminished production and expression of Th1 cytokines, including IFN- $\gamma$ and IL-2 [4]. Additionally, this same report demonstrated that after 9 months of drug therapy, IFN- $\gamma$ levels were markedly increased, coinciding with successful treatment. As a result, it has been suggested that different clinical states of Mtb can be correlated with cytokine profiles.

In an effort to reduce the amount of time required to accurately diagnose an Mtb infection, some attention is being focused on identifying biomarkers of Mtb infections; examples include molecules that are secreted by the pathogen itself, or host factors derived from immune responses. In this study, we build upon our previous findings that TNF- $\alpha$, IFN- $\gamma$ and IL-10 profiles are distinct in non-MDR-TB versus MDR-TB patients [5]. Using sputum clearance as an indicator of positive responses to chemotherapies, we report that patients on anti-Mtb chemotherapies have altered TNF- $\alpha$ and IFN- $\gamma$ levels that correlate with favorable responses to the drugs. As a result, we propose that these cytokines, particularly TNF$\alpha$, could serve as potential biomarkers for response to therapy.

\section{Patients and Methods}

\section{Study Subjects}

A total of 39 patients ( 35 men and 4 women) who were diagnosed with active TB by clinical findings, radiological examination and the demonstration of acid-fast bacilli (AFB) in their sputum at the National Masan TB Hospital (NMTH) were en- 
rolled for this study (table 1). Thirty-one bacillus CalmetteGuérin (BCG)-vaccinated, healthy individuals (15 men and 16 women) also participated as controls. The study protocol was approved by the NMTH Institutional Ethics Committee and written informed consent was obtained from each study subject.

Sputum Culture and Drug Susceptibility Test

Sputum culture positivity for Mtb was confirmed both by BacT/Alert and conventional Ogawa media. Briefly, sputum specimens were decontaminated with $\mathrm{N}$-acetyl-L-cysteine/4\% $\mathrm{NaOH}$ and inoculated into BacT/Alert and Ogawa media. When the Ogawa growth was detected as positive, a drug susceptibility test was carried out on Löwenstein-Jensen plates containing anti-TB drugs. The 'absolute concentration method' was used as previously described $[6,7]$. The critical concentration for isoniazid and rifampin was 0.2 and $40 \mu \mathrm{g} / \mathrm{ml}$, respectively. All inoculated slants were incubated at $37^{\circ} \mathrm{C}$ and growth was checked visually every week. The sputum culture staining for AFB was assessed at the time of admission, and 2 and 6 months after starting proper treatment.

\section{Blood Collection and Cytokine Whole-Blood Assay}

Blood samples were drawn into heparinized Vacutainers and processed within $2 \mathrm{~h}$ for the cytokine whole-blood assay [5, 8]. Blood was collected at the time of admission, and 2 and 6 months after the start of treatment prescribed by NMTH staff. Blood diluted with RPMI-1640 (1:10) containing penicillin-streptomycin (Gibco) and 10\% fetal bovine serum was distributed into 96-well plates and cultured in triplicate in the presence of medium alone or with phytohemaggultinin (PHA) or lipopolysaccharide (LPS; Sigma-Aldrich, St. Louis, Mo., USA) or culture filtrate protein (CFP) at a final concentration of $10 \mu \mathrm{g} / \mathrm{ml}$. Cultures were maintained in a $5 \% \mathrm{CO}_{2}$ atmosphere at $37^{\circ} \mathrm{C}$. Cultured supernatants were harvested 1,3 and 6 days after culturing, and stored at $-70^{\circ} \mathrm{C}$ until use. Cytokines were measured at day 1 for IL-12 and IL-10, day 3 for TNF- $\alpha$ and day 6 for IFN- $\gamma$ (optimal time points for each cytokine were determined by preliminary experiments, data not shown).

\section{Measurement of Cytokines by ELISA}

For cytokine analysis, two-site capture immunoassays with OptEIA monoclonal antibody sets (BD Pharmingen, Los Angeles, Calif., USA), streptavidin-horseradish peroxidase conjugate and recombinant cytokines as standards were used. Briefly, 96-well plates were coated with capture antibodies to each cytokine (IFN$\gamma$, IL-12, TNF- $\alpha$ and IL-10) according to the manufacturer's protocol. The concentrations were given in the ELISA set instructions as dilution factor, 1:250. Plates were incubated overnight at $4{ }^{\circ} \mathrm{C}$ and blocked at room temperature with 5\% FBS in PBS for $1 \mathrm{~h}$. The plates were washed three times. Samples were added and incubated for $2 \mathrm{~h}$ at room temperature. Working detector (detection antibody + avidin-HRP reagent) was added to each well and the plate was incubated for $1 \mathrm{~h}$ at room temperature. After washing 7 times, substrate solution (BD Pharmingen TMB substrate reagent set) was added to each sample. The plates were incubated for $30 \mathrm{~min}$ and the reaction was stopped by adding stop solution. The plates were read at $450 \mathrm{~nm}$ in an ELISA reader (OPTImax, Sunnyvale, Calif., USA). A standard curve was generated from the known concentration of the standard according to the method provided in the kit. Cytokine levels were calculated from the standard curve by computing absorbance values against the test concentration. Detection limits of the cytokine kits used were as follows: IFN- $\gamma-5 \mathrm{pg} / \mathrm{ml}$; IL-12 - $7.5 \mathrm{pg} / \mathrm{ml}$; TNF- $\alpha-15 \mathrm{pg} / \mathrm{ml}$, and IL-10 $-7.5 \mathrm{pg} / \mathrm{ml}$.

\section{Statistical Analysis}

The Mann-Whitney U test was used for data analysis between groups. Differences were considered significant when $\mathrm{p} \leq 0.05$.

\section{Results}

\section{Patient Description and Response to Chemotherapy}

Thirty-nine subjects were enrolled in this study (table 1). All subjects were examined for clinical symptoms and underwent standard radiographic assessment. The mean age of the patients was $49.4 \pm 2$ years (range, $22-87$ years). They included 14 newly diagnosed, 6 relapse, 7 chronic, 6 treatment failure, and 6 default cases as defined by the World Health Organization [9]. One case of pneumothorax was observed and 11 subjects had concomitant diabetes mellitus, and 1 had hypertension. None of the patients were HIV positive. At the time of initial blood sampling, the patients had been started on chemotherapy for $<1$ week. Thirty-one Microbacterium bovis BCG-vaccinated, healthy individuals (15 men and 16 women) also participated as controls, and their mean age was $34.6 \pm 1.5$ years (range, 23-54 years). Of the $39 \mathrm{~TB}$ patients, 10 were culture-confirmed non-MDR-TB while 16 were culture-confirmed MDR-TB patients. Thirteen of the initial patients were sputum culture negative at the start of the therapy, and remained so throughout the 6month study. Although they were culture negative, 12 of 13 of these patients experienced significant clinical improvement with normal first-line chemotherapy during the course of this study while 1 patient did not. For that reason, this non-responsive patient was omitted from further analysis. In total, therefore, 38 patients were considered to have diagnosed as TB and were included in subsequent analysis: 22 had drug-susceptible disease and 16 MDR disease.

Of the 10 sputum-positive drug-susceptible subjects, 9 had achieved sputum conversion after the first 2 months of therapy, and the last subject achieved sputum conversion after 6 months. In total, 100\% of the non-MDR-TB subjects were sputum negative at the end of the therapy. Of the 16 MDR-TB subjects, only 2 subjects had achieved sputum conversion after 2 months. After 6 months of treatment, an additional 6 subjects ( 8 in total) had converted. In total, $50 \%$ of the MDR-TB patients had con- 


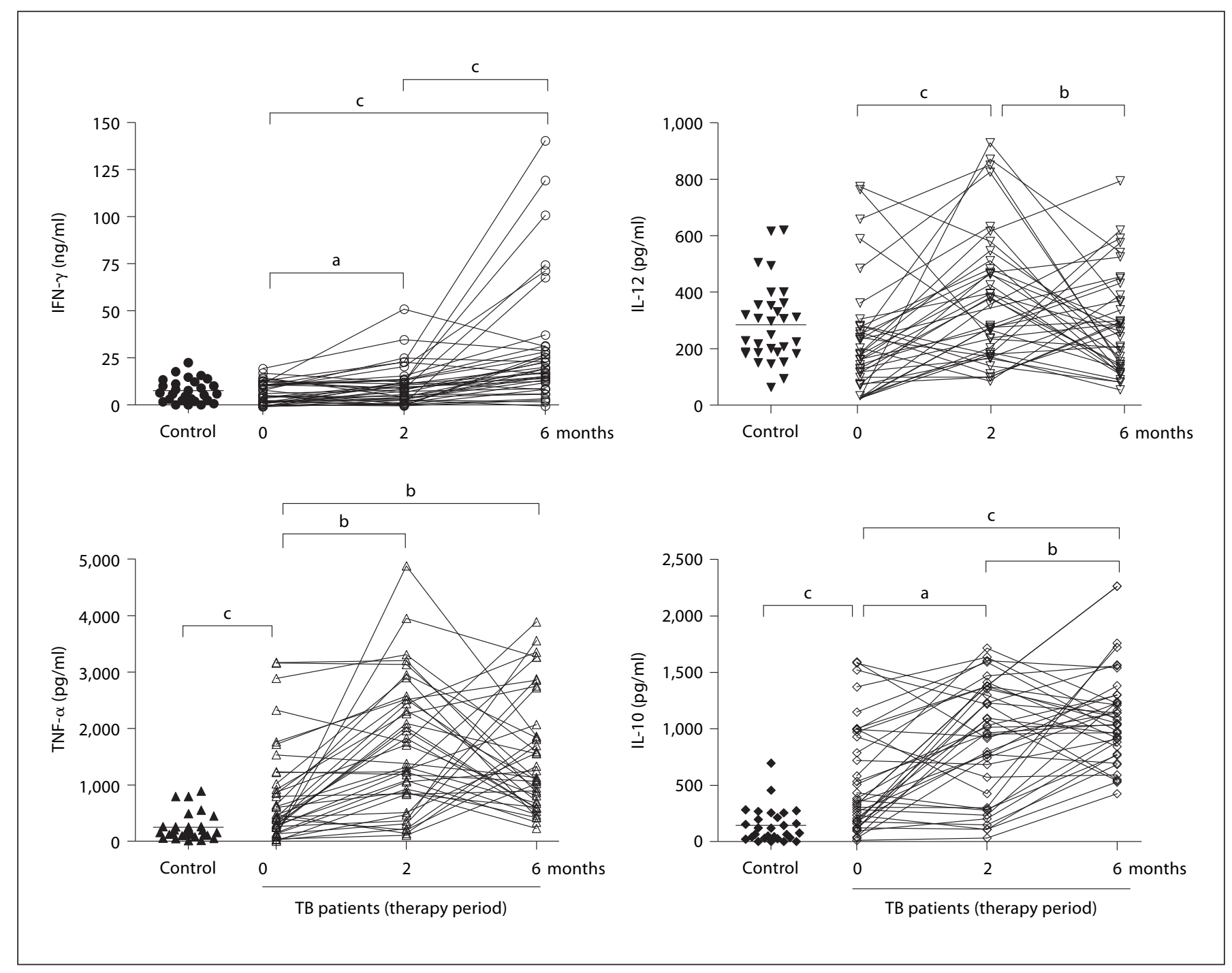

Fig. 1. Cytokine profiles and modulation by chemotherapy in whole blood from TB patients in comparison to healthy controls. Whole blood was incubated with CFP $(10 \mu \mathrm{g} / \mathrm{ml})$ for 1,3 or 6 days and cytokine concentrations were assessed by ELISA. Each patient is represented by entry, and 2- and 6-month measurements (the mean of triplicate cultures after deducting the medium alone value). ${ }^{\mathrm{a}} \mathrm{p} \leq 0.05,{ }^{\mathrm{b}} \mathrm{p} \leq 0.01$ and ${ }^{\mathrm{c}} \mathrm{p} \leq 0.001$.

verted to sputum negative by the conclusion of the study (table 1).

\section{Whole-Blood Cytokine Profiles during Chemotherapy}

To explore whether changes in cytokine responses before, during and after 6 months of drug therapy would provide a surrogate marker for sputum smear and culture, we collected whole blood from healthy volunteers $(\mathrm{n}=31)$ and our cohort of TB patients. This blood was stimulated with CFP from Mtb and 4 cytokines were measured: IFN- $\gamma$, TNF- $\alpha$, IL-10 and IL-12 (fig. 1).
At the start of treatment, there was no significant difference in the IFN- $\gamma$ and IL-12 levels between TB patients and healthy controls. IFN- $\gamma$ levels increased with therapy by 2 months $(\mathrm{p} \leq 0.05)$ and reached a plateau almost threefold higher compared to levels before treatment by 6 months of therapy. IL-12 levels similarly increased after 2 months of therapy ( $\mathrm{p} \leq 0.01$ ) but decreased to levels before therapy by 6 months (fig. 1). In contrast, significantly higher levels of TNF- $\alpha$ and IL-10 were observed in TB patients than healthy controls at the start of the treatment, and these cytokine levels also increased during 


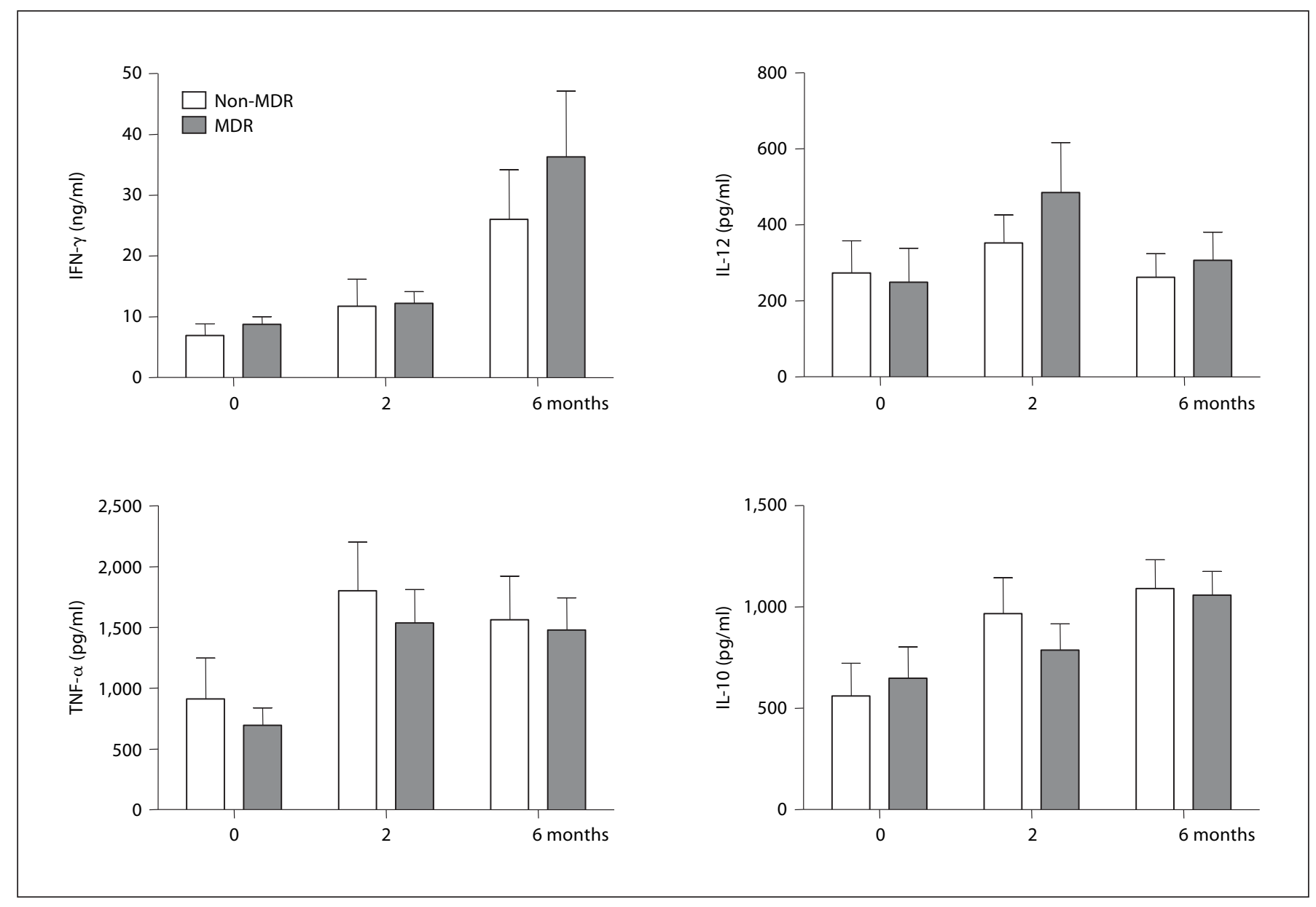

Fig. 2. Comparison of cytokine production and modulation by chemotherapy between non-MDR and MDR-TB patients. Whole blood was incubated with CFP $(10 \mu \mathrm{g} / \mathrm{ml})$ for 1,3 or 6 days and cytokine concentrations were assessed by ELISA. Each value was calculated as the mean of triplicate cultures after deducting the medium alone value. Results are represented as means \pm SEM.

drug treatment (fig. 1). In general, all 4 cytokines showed a complex dynamic response that appeared to fall into one of two general trends: either a transient increase at 2 months followed by a return to baseline levels at 6 months, or an increase at 2 months followed by a further increase at 6 months.

\section{Cytokine Response Pattern Does Not Correlate with MDR Status But Is Mtb Specific}

In an attempt to understand whether these two patterns of response were the result of previous treatment history or were specific to strains of Mtb that had drug resistance, we divided the patients into non-MDR- and MDR-TB groups and examined patterns of cytokine production between these groups, but no significant differ- ences were observed (fig. 2). Likewise, when comparing sputum culture-negative subjects that showed clinical improvement to sputum culture-positive patients, no differences in responses of cytokine profiles were observed (data not shown).

In order to verify that the cytokine responses seen were in fact specific to the Mtb antigens present in CFP, we stimulated the same blood samples with non-TB-related mitogens to activate the aforementioned cytokines. For these purposes, PHA was used to stimulate IFN- $\gamma$ and IL-10, while LPS was used for TNF- $\alpha$ and IL-12 stimulation (fig. 3). No changes in IFN- $\gamma$ levels were observed between these groups during therapy. Similar results were obtained with TNF- $\alpha$ and IL-12 when comparing CFP and mitogen stimulation. Interestingly, PHA-in- 


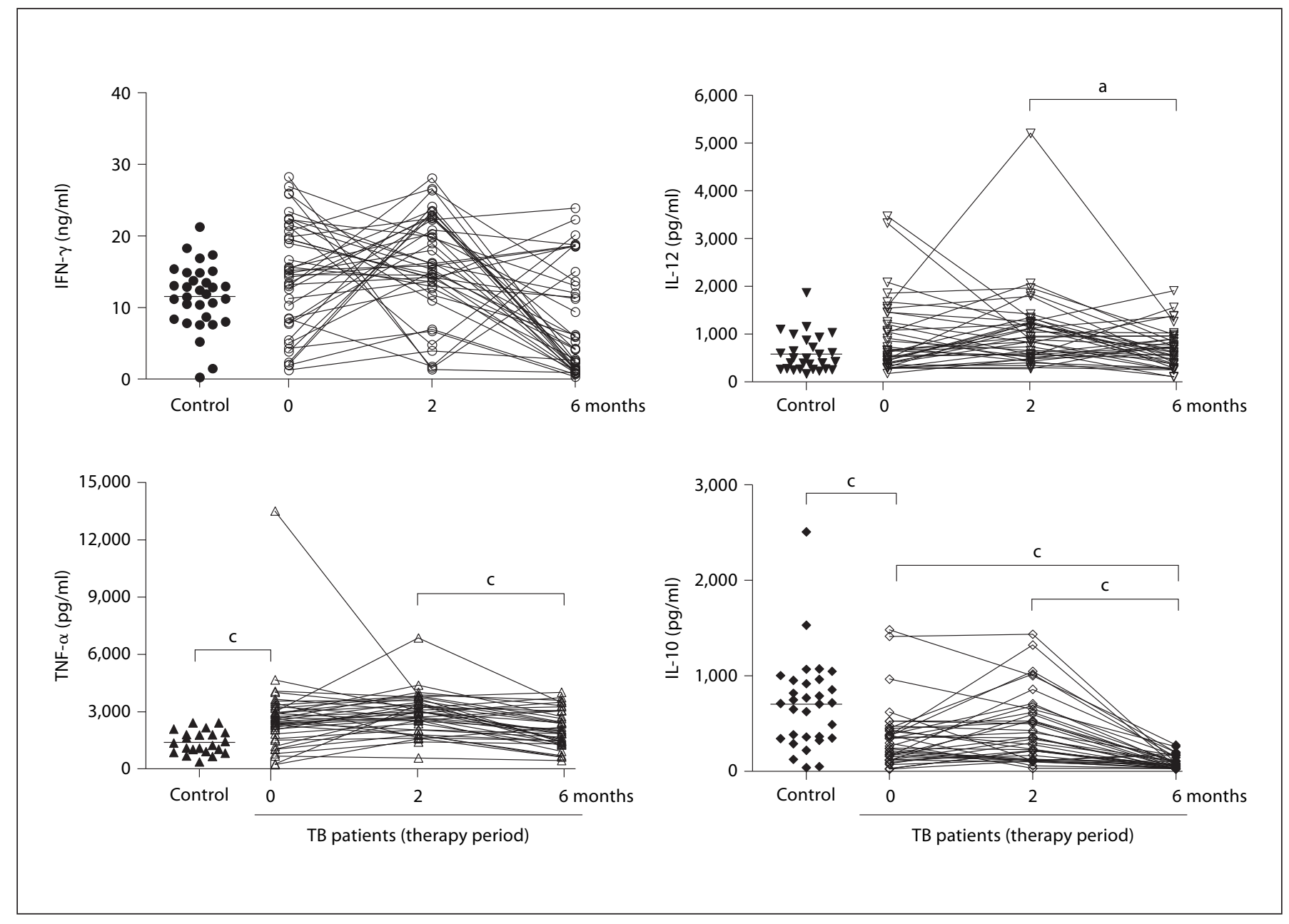

Fig. 3. Cytokine profiles in whole blood after stimulation with PHA (IFN- $\gamma$ and IL-10) or LPS (IL-12 and TNF$\alpha)$ from TB patients and healthy controls. Each patient is represented by entry, and 2- and 6-month measurements (the mean of triplicate cultures after deducting medium alone value). ${ }^{\mathrm{a}} \mathrm{p} \leq 0.05$ and ${ }^{\mathrm{c}} \mathrm{p} \leq 0.001$.

duced IL-10 production showed a reverse correlation with PHA induction (fig. 3).

\section{Effect of Sputum Clearance Rate on Cytokine \\ Production Patterns}

In an effort to see if the cytokine production patterns correlated with sputum conversion, we obtained sputum cultures from each subject at the time of blood drawing for cytokine assays and used these results to identify the sputum-positive or sputum-negative status at each time point (fig. 4). Prior to the start of therapy, IFN- $\gamma$ levels were overall lower in the 12 sputum culture-negative subjects compared to the culture-positive subjects, a trend that continued when comparing groups of patients who had achieved a culture-negative status compared to those who remained culture positive at each time point throughout the study. At the end of the treatment, those who remained culture positive had dramatically increased amounts of IFN- $\gamma$ compared to levels both before the therapy and after 2 months even compared to those who had converted at 6 months. A similar pattern to IFN- $\gamma$ levels was seen with IL-12. Lower levels of IL-12 were released in the patients who had converted to culture negative than those who failed to at every time point. This result was statistically significant $(\mathrm{p}<0.05)$ after 6 months of therapy (fig. 4).

TNF- $\alpha$ and IL-10 showed somewhat different patterns than IFN- $\gamma$ and IL-12 in this cohort. Prior to therapy, sputum-positive and sputum-negative subjects had similar levels of TNF- $\alpha$ and IL-12. Although statistically sim- 


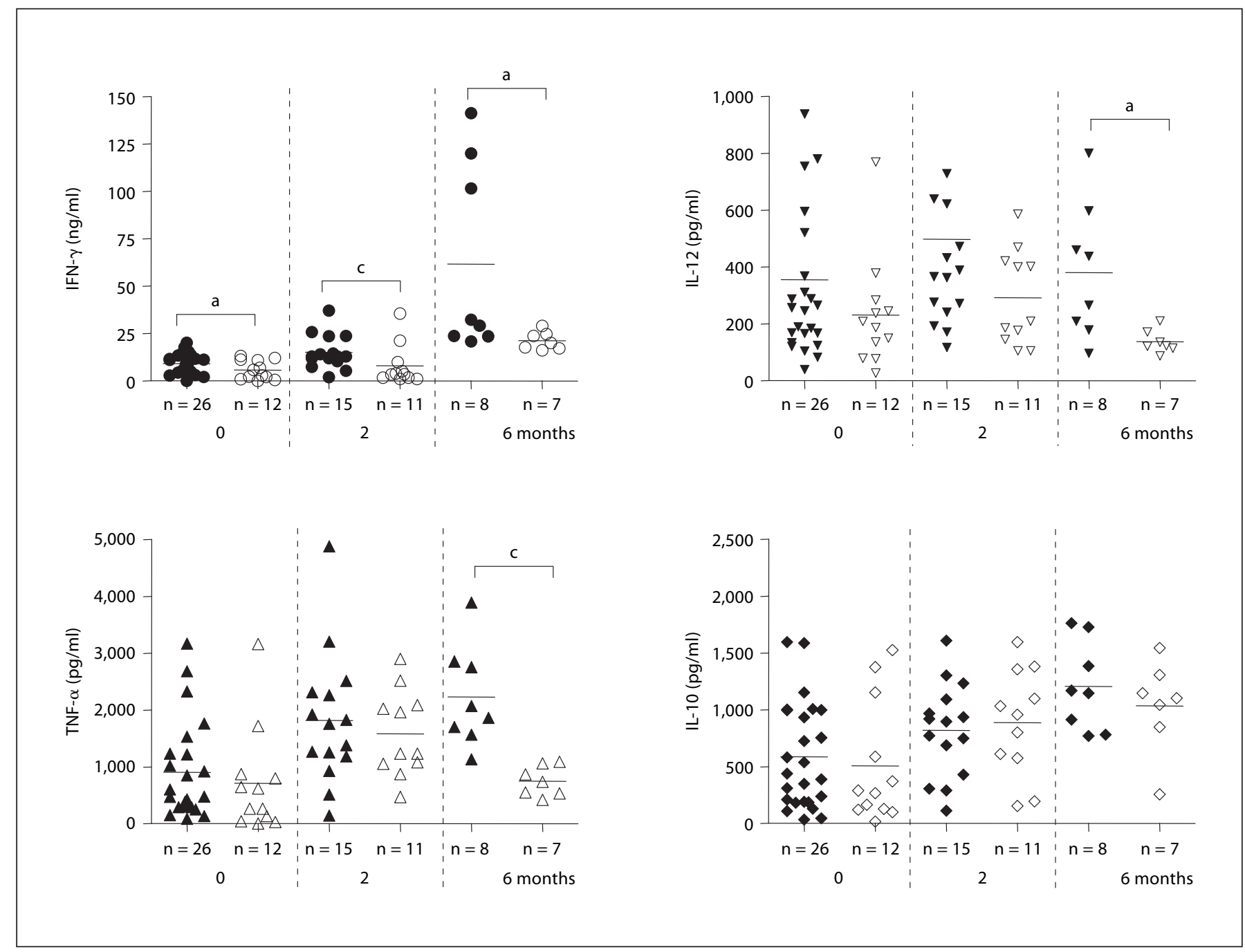

Fig. 4. Comparison of cytokine production patterns between culture-positive (black) and -negative (empty) patients. Sputum cultures were performed at each time point and cytokine production was compared between culture-positive (black) and -negative (empty) patients. Only those patients who were sputum culture positive at the previous time point were reevaluated at the next time point of therapy. Therefore, only 26 sputum-positive patients were evaluated at 2 months and only 15 sputum-positive patients were evaluated at 6 months. Each value was calculated as the mean of triplicate cultures after deducting medium alone value. ${ }^{\mathrm{a}} \mathrm{p} \leq$ 0.05 and ${ }^{\mathrm{c}} \mathrm{p} \leq 0.001$. ilar, both groups doubled their levels at 2 months. However, a dramatic change in TNF- $\alpha$ was seen at 6 months, when the sputum-negative population returned to cytokine levels before the therapy while remaining elevated in the sputum-positive population $(\mathrm{p}<0.001)$. Strikingly, IL-10, a cytokine which is involved in helping to turn off the immune response, was relatively unchanged by the presence or absence of Mtb in the sputum of subjects before, during or after the therapy.

TNF- $\alpha$ as Response to Chemotherapy in Tuberculosis

\section{Discussion}

Cytokine production patterns in TB patients are presumed to vary according to the disease state, and we have previously reported that TNF- $\alpha$ was associated with MDR-TB and mycobacterial load in sputum [5]. However, our previous study was cross-sectional, limiting our ability to evaluate these correlations as markers to potentially predict outcome. In this longitudinal study, we have extended this observation by comparing mycobacterial 
clearance during chemotherapy with TNF- $\alpha$ levels produced by stimulated whole blood in the same patients over time.

Prior to starting the therapy, TNF- $\alpha$ and IL-10 levels were significantly higher in TB patients than in healthy controls. These results are in agreement with other published results for IL-10 $[10,11]$ and for TNF- $\alpha$ [12-14]. In contrast, IFN- $\gamma$ and IL-12 levels were not different between healthy controls and TB patients. Many other studies report that the production of IFN- $\gamma$ is depressed in TB patients compared to healthy individuals [15-17]. However, conflicting reports have demonstrated no differences in IFN- $\gamma$ mRNA expression and the number of IFN$\gamma$-producing cells between TB patients and healthy controls $[18,19]$. This discrepancy may be explained by recent studies reporting that different disease states could induce different IFN- $\gamma$ responses [17, 20, 21]. In our patient cohort, during anti-TB chemotherapy, the levels of IFN- $\gamma$ increased significantly after 2 months and much more after 6 months in TB patients, consistent with other reports $[4,8]$. IL-12 also increased after 2 months of therapy, but after 6 months of therapy decreased again to levels before therapy, whereas IFN- $\gamma$ showed the highest production levels at 6 months. Although IL-12 is known to enhance IFN- $\gamma$ production $[2,22]$, this observation suggests IFN- $\gamma$ production is not dependent on IL-12 regulation, as already shown in another study $[23,24]$. The decrease in IL-12 production after 6 months of therapy may be related to the increase in the anti-inflammatory cytokine IL-10. IL-10 is known to be produced by macrophages and $\mathrm{T}$ cells during Mtb infection and possesses macrophage-deactivating properties including downregulating IL-12 production [25]. Because IL-10 acts as an antagonist of TNF- $\alpha$ and IFN- $\gamma$, it appears contradictory that IFN- $\gamma$ and TNF- $\alpha$ would increase concomitantly with IL-10. However, it is plausible to consider that IL-10 is functioning solely to downregulate the immune system. As drug treatment clears Mtb from the host, the need for IFN- $\gamma$ and TNF- $\alpha$ production decreases, an event regulated by IL-10. It is also in agreement with the drop in IL-12 to levels before the treatment, further indicating a reduction in the immune response.

IFN- $\gamma$ is also involved in stimulating TNF- $\alpha$ secretion [26]. This study shows the induction of TNF- $\alpha$ increased as IFN- $\gamma$ production increased during the course of chemotherapy. Mitogen-induced cytokines showed different profiles from CFP-induced cytokines. No changes in levels were observed in PHA-induced IFN- $\gamma$ production with therapy but LPS-induced IL-12 and TNF- $\alpha$ production levels correlated with those of CFP-induced levels. In con- trast, PHA-induced IL-10 showed an inverse correlation with CFP-induced IL-10 production. The molecular and kinetic basis for this difference in response is not clear, however, successful therapy may have repressed the production of this immunosuppressive cytokine by non-specific stimulation. Our findings suggest that chemotherapy may alter the specific cytokine production profiles.

In order to investigate if mycobacterial clearance induced by chemotherapy is mirrored by alterations in the cytokine response, serial sputum culture and cytokine measurements during therapy were examined. Almost all non-MDR-TB patients (9/10) who were initially sputum culture positive became sputum culture negative after the first 2 months of therapy, whereas only 2 MDR-TB patients obtained sputum negativity. At this time point, both IFN- $\gamma$ and IL-12 production were decreased more in sputum-negative patients than in sputum-positive patients, indicating the normalization of the production of these 2 cytokines. However, no differences between patient groups were observed in TNF- $\alpha$ and IL-10 levels. By 6 months after the therapy, 6 further MDR-TB patients had become sputum culture negative, and IFN- $\gamma$ and IL12 production also decreased in these sputum-negative patients compared to the persistently sputum-positive patients. Notably, IL-10 levels remained unchanged between the two groups. However, there was a marked reduction in TNF- $\alpha$ to levels similar to those seen in the group of sputum-negative patients before the therapy. These findings suggest the potential role of TNF- $\alpha$ as a marker for successful therapy in MDR-TB.

It has already been demonstrated that some MDR-TB patients produce lower levels of TNF- $\alpha$ but not IFN- $\gamma$ compared with non-MDR-TB patients in PBMCs [27] or in whole blood [5]. Also, antigenic stimulation of peripheral blood monocytes from MDR-TB induces different trends of TNF- $\alpha$ and IL-12 production compared to those from non-MDR-TB patients [27]. The production of nitric oxide from peripheral blood monocytes was depressed in MDR-TB patients and correlated with TNF- $\alpha$ production [28]. Conversely, sputum TNF- $\alpha$ levels decrease following initiation of treatment [29] and a positive correlation was observed between mycobacterial load and TNF- $\alpha$ levels in sputum by 12 weeks of treatment [29]. While this may reflect variation in the patient populations studied it is worth at least considering that this may reflect differential antigenicity of the TB strains that are more prone to develop into MDR disease. It is worth pointing out that at least one virulence factor of Beijing strains (the phenolic glycolipid) has been shown to have potent immunomodulatory properties [30]. 
TNF- $\alpha$ appears to be the most dynamic of the 4 cytokines investigated here, a finding that should support further evaluation of the response of this cytokine in TB patients. Our results suggest CFP-induced TNF- $\alpha$ levels in whole blood are closely related to the clearance of $\mathrm{Mtb}$ in MDR-TB patients independent of IL-10 regulation and could be a useful indicator for monitoring response to therapy in MDR-TB patients. However, the sample size in the present study is too small to make a definitive conclusion and this hypothesis will have to be validated in larger patient cohorts. These studies also suggest the possibility that an immune signature that correlates with sputum conversion may exist that would provide an early indicator for discontinuation of therapy, a significant problem for MDR-TB where treatment courses often exceed 2 years.

\section{Acknowledgments}

This study was supported in part by the Division of Intramural Research, National Institute of Allergy and Infectious Diseases, National Institutes of Health, Bethesda, Md., USA, and by a grant from the Brain Korea 21 Project for Medical Sciences in Yonsei University (S.-N.C.).

\section{References}

1 Blasi F, Tarsia P, Aliberti S: Strategic targets of essential host-pathogen interactions. Respiration 2005;72:9-25.

-2 Flynn JL, Chan J: Immunology of tuberculosis. Ann Rev Immunol 2001;19:93-129.

$\checkmark 3$ Vergne I, Chua J, Deretic V: Tuberculosis toxin blocking phagosome maturation inhibits a novel $\mathrm{Ca}^{2+} /$ calmodulin-PI3K hVPS34 cascade. J Exp Med 2003;198:653-659.

$\checkmark 4$ Zhang M, Lin Y, Iyer DV, Gong J, Abrams JS, Barnes PF: T-cell cytokine responses in human infection with Mycobacterium tuberculosis. Infect Immun 1995;63:3231-3234.

5 Eum SY, Jeon BY, Min JH, Kim SC, Cho SA, Park SK, Cho SN: Tumor necrosis factoralpha and interleukin-10 in whole blood is associated with disease progression in pulmonary multidrug-resistant tuberculosis patients. Respiration 2008;76:331-337.

-6 Canetti G, Fox W, Khomenko A, Mahler HT, Menon NK, Mitchison DA, Rist N, Smelev NA: Advances in techniques of testing mycobacterial drug sensitivity, and the use of sensitivity tests in tuberculosis control programmes. Bull World Health Organ 1969;41: 21-43.

7 Heifets LB: Drug Susceptibility in the Chemotherapy of Mycobacterial Infections. Boca Raton, CRC, 1991, pp 89-121.

$>8$ Hussain R, Talat N, Shahid F, Dawood G: Longitudinal tracking of cytokines after acute exposure to tuberculosis: association of distinct cytokine patterns with protection and disease development. Clin Vaccine Immunol 2007;14:1578-1586.

>9 World Health Organization, International Union against Tuberculosis and Lung Disease, Royal Netherlands Tuberculosis Association: Revised international definitions in tuberculosis control. Int J Tuberc Lung Dis 2001;5:213-215.
10 Torres M, Herrera T, Villareal H, Rich EA, Sada E: Cytokine profiles for peripheral blood lymphocytes from patients with active pulmonary tuberculosis and healthy household contacts in response to the 30-kilodalton antigen of Mycobacterium tuberculosis. Infect Immun 1998;66:176-180.

$>11$ Lee JS, Song CH, Kim CH, Kong SJ, Shon MH, Kim HJ, Park JK, Paik TH, Jo EK: Profiles of IFN-gamma and its regulatory cytokines (IL-12, IL-18 and IL-10) in peripheral blood mononuclear cells from patients with multidrug-resistant tuberculosis. Clin Exp Immunol 2002;128:516-524.

$>12$ Hirsch CS, Toossi Z, Othieno C, Johnson JL, Schwander SK, Robertson S, Wallis RS, Edmonds K, Okwera A, Mugerwa R, Peters P, Ellner JJ: Depressed T-cell interferon- $\lambda$ responses in pulmonary tuberculosis: analysis of underlying mechanisms and modulation with therapy. J Infect Dis 1999; 180:20692073.

13 Ogawa T, Uchida H, Kusumoto Y, Mori Y, Yamamura Y, Hamada S: Increase in tumor necrosis factor alpha- and interleukin-6-secreting cells in peripheral blood mononuclear cells from subjects with Mycobacterium tuberculosis. Infect Immun 1991;59:30213025 .

-14 Kupeli E, Karnak D, Beder S, Kayacan O, Tutkak H: Diagnostic accuracy of cytokine levels (TNF- $\alpha$, IL- 2 and IFN- $\gamma$ ) in bronchoalveolar lavage fluid of smear-negative pulmonary tuberculosis patients. Respiration 2008;75:73-78.

15 Hussain R, Kaleem A, Shahid F, Dojki M, Jamil B, Mehmood H, Dawood G, Dockrell HM: Cytokine profiles using whole-blood assays can discriminate between tuberculosis patients and healthy endemic controls in a BCG-vaccinated population. J Immunol Methods 2002;264:95-108.
16 Sanchez FO, Rodriguez JI, Agudelo G, Garcia LF: Immune responsiveness and lymphokine production in patients with tuberculosis and healthy controls. Infect Immun 1994; 62:5673-5678.

17 Inokuchi N, Sugahara K, Soda H, Usui T, Hirakata Y, Fukushima K, Yamada Y, Kohno S, Kamihira S: Relationship between wholeblood interferon-gamma production and extent of radiographic disease in patients with pulmonary tuberculosis. Diagn Microbiol Infect Dis 2003;46:109-114.

18 Johnson BJ, McMurray DN: Cytokine gene expression by cultures of human lymphocytes with autologous Mycobacterium tuberculosis-infected monocytes. Infect Immun 1994;62:1444-1450.

19 Surcel HM, Troye-Blomberg M, Paulie S, Andersson G, Moreno C, Pasvol G, Ivanyi J: Th1/Th2 profiles in tuberculosis, based on the proliferation and cytokine response of blood lymphocytes to mycobacterial antigens. Immunology 1994;81:171-176.

20 Ellner JJ, Hirsch CS, Whalen CC: Correlates of protective immunity to Mycobacterium tuberculosis in humans. Clin Infect Dis 2000; 30:S279-S282.

21 Sodhi A, Gong J, Silva C, Qian D, Barnes PF: Clinical correlates of interferon- $\gamma$ production in patients with tuberculosis. Clin Infect Dis 1997;25:617-620.

22 Cooper AM, Magram J, Ferrante J, Orme IM: Interleukin 12 (IL-12) is crucial to the development of protective immunity in mice intravenously infected with Mycobacterium tuberculosis. J Exp Med 1997;186:39-45. 
-23 Sahiratmadja E, Alisjahbana B, de Boer T, Adnan I, Maya A, Danusantoso H, Nelwan RHH, Marzuki S, van der Meer JWM, van Crevel R, van de Vosse E, Ottenhoff THM: Dynamic changes in pro- and anti-inflammatory cytokine profiles and gamma interferon receptor signaling integrity correlate with tuberculosis disease activity and response to curative treatment. Infect Immun 2007;75:820-829.

24 Lee HW, Lee HS, Kim DK, Ko DS, Han SK, Shim YS, Yim JJ: Lack of association between interleukin-12 receptor $\beta 1$ polymorphisms and tuberculosis in Koreans. Respiration 2005;72:365-368.
25 Gong JH, Zhang M, Modlin RL, Linsley PS, Iyer D, Lin Y, Barnes PF: Interleukin-10 downregulates Mycobacterium tuberculosisinduced Th1 responses and CTLA- 4 expression. Infect Immun 1996;64:913-918.

26 Appelberg R, Castro AG, Pedrosa J, Silva RA, Orme IM, Minoprio P: Role of gamma interferon and tumor necrosis factor alpha during T-cell-independent and -dependent phases of Mycobacterium avium infection. Infect Immun 1994;62:3962-3971.

27 Lee JS, Song CH, Lim JH, Kim HJ, Park JK, Paik TH, Kim CH, Kong SJ, Shon MH, Jung SS, Jo EK: The production of tumor necrosis factor-alpha is decreased in peripheral blood mononuclear cells from multidrug-resistant tuberculosis patients following stimulation with the $30-\mathrm{kDa}$ antigen of Mycobacterium tuberculosis. Clin Exp Immunol 2003;132: 443-449.
28 Sharma S, Sharma M, Roy S, Kumar P, Bose $\mathrm{M}$ : Mycobacterium tuberculosis induces high production of nitric oxide in coordination with production of tumor necrosis factor-alpha in patients with fresh active tuberculosis but not in MDR tuberculosis. Immunol Cell Biol 2004;82:377-382.

29 Ribeiro-Rodrigues R, Resende Co T, Johnson JL, Ribeiro F, Palaci M, Sa RT, Maciel EL, Pereira Lima FE, Dettoni V, Toossi Z, Boom WH, Dietze R, Ellner JJ, Hirsch CS: Sputum cytokine levels in patients with pulmonary tuberculosis as early markers of mycobacterial clearance. Clin Diagn Lab Immunol 2002;9:818-823.

30 Reed MB, Domenech P, Manca C, Su H, Barczak AK, Kreiswirth BN, Kaplan G, Barry CE 3rd: A glycolipid of hypervurulent tuberculosis strains that inhibits the innate immune response. 2004;431:84-87. 